\title{
"Naked" gold nanoparticles supported on HOPG: melanin functionalization and catalytic activity
}

\author{
A. González Orive, ${ }^{b}$ D. Grumelli, ${ }^{* a}$ C. Vericat, ${ }^{a}$ J. M. Ramallo-López,${ }^{a}$ L. Giovanetti, ${ }^{a}$ G. Benitez, ${ }^{a}$ \\ J. C. Azcárate, ${ }^{a}$ G. Corthey, ${ }^{a}$ M. H. Fonticelli, ${ }^{a}$ F. G. Requejo, ${ }^{a}$ A. Hernández Creus ${ }^{b}$ and R. C. Salvarezza ${ }^{a}$
}

Received 27th November 2010, Accepted 10th January 2011

DOI: 10.1039/c0nr00911c

Reductive electrodesorption has been used to produce "naked" gold nanoparticles (AuNPs) $3 \mathrm{~nm}$ in size on HOPG from different thiolate-capped AuNPs. The clean AuNPs transform the electrocatalytic inert HOPG into an active surface for hydrogen peroxide electroreduction, causing a lowering of the cathodic overpotential of $0.25 \mathrm{~V}$ with respect to the $\mathrm{Au}(111)$ surface. Compared to the plain gold substrates, the nanostructures promote only a slight increase in the hydrogen evolution reaction. In a second modification step a $\sim 1 \mathrm{~nm}$ thick melanin-iron coating is electrochemically formed around the AuNPs. This ultrathin melanin-iron coating largely improves the catalytic activity of the bare AuNPs for both hydrogen peroxide electroreduction and hydrogen evolution reaction. This strategy, which integrates electrochemistry and nanotechnology, can be applied to the preparation of efficient "naked" AuNPs and organic-iron capped AuNPs catalysts.

\section{Introduction}

The preparation of bare nanometre sized metallic nanoparticles (NPs) with a narrow size distribution on solid surfaces is an active field of research in nanoscience and nanotechnology, with particular interest in heterogeneous catalysis and electrocatalysis. ${ }^{1,2}$ Among metallic nanoparticles, gold nanoparticles (AuNPs) have attracted considerable attention since the discovery that they are able to catalyze oxidation, hydrogenation and hydrochlorination reactions when supported by oxides or carbon substrates. ${ }^{3-8}$ In general, in order to have excellent catalytic activities gold nanoparticles $1-5 \mathrm{~nm}$ in diameter with a narrow size distribution are required..$^{9}$

For catalytic purposes, metallic nanoparticles can be usually prepared either by co-deposition of a Au salt and a metal oxide, ${ }^{10}$ by deposition-precipitation using precipitation agents, by cosputtering of gold and metal oxide, or by chemical vapor deposition of gold nanoparticles. ${ }^{3}$ However, AuNPs prepared by these methods usually exhibit a size distribution too broad to have defined size-dependent properties. Better quality metallic nanoparticles can be produced by wet chemistry synthetic methods. ${ }^{11,12}$ However, these methods require the use of organic stabilizers such as thiols, which in most cases must be completely removed in order to use the NPs as efficient catalysts and

${ }^{a}$ Instituto de Investigaciones Fisicoquímicas Teóricas y Aplicadas (INIFTA), Facultad de Ciencias Exactas, Universidad Nacional de La Plata-CONICET, Sucursal 4 Casilla de Correo 16, 1900 La Plata, Argentina.E-mail: doris@inifta.unlp.edu.ar; Fax: +54-221-4254642; Tel: +54-221-4257291/4257430

${ }^{b}$ Departamento de Química Física, Universidad de La Laguna, Avda. Astrofísico Francisco Sanchez S/N, La Laguna, 38071, Tenerife, Spain electrocatalysts. Different methods are available to remove the thiol components from thiolated surfaces, like thermal treatment, UV-exposure and atomic hydrogen, ${ }^{13}$ and all of them present both advantages and disadvantages. Finally, the nanoparticles prepared by wet chemistry must be efficiently immobilized on a solid support in order to be employed in heterogeneous catalysis and electrocatalysis. However, clean AuNPs on external surfaces are susceptible to aggregation and sintering, thus reducing their catalytic properties.

Therefore, the application of AuNPs capped by molecules in catalysis and electrocatalysis requires the ability to manipulate the core and the capping properties in controllable ways. In this context, the development of new strategies capable of immobilizing, cleaning and functionalizing stable small metallic NPs by using simple and inexpensive methods is a frontier topic that deserves special attention.

We have recently demonstrated that butanethiol-capped AuNPs prepared by the Brust method can be adsorbed on highly oriented pyrolytic graphite (HOPG) by simple immersion in AuNP containing solutions. ${ }^{14}$ The immobilized AuNPs substrate can be subsequently cleaned by reductive desorption of the short thiol, leading to bare nanometre sized NPs on the carbon substrates.

In this work electrochemistry is used to produce bare $\sim 3 \mathrm{~nm}$ AuNPs on HOPG surfaces starting from nonanethiol (NT)- and dodecanethiol (DT)-capped AuNPs prepared by the Brust method. We demonstrate that even relatively long thiols, for which hydrocarbon-hydrocarbon chain interactions are more important, can be completely removed from the AuNPs by reductive desorption. The bare nanoparticles increase the electrocatalytic activity for hydrogen peroxide electroreduction and 
for the hydrogen evolution reaction with respect to the $\mathrm{Au}(111)$ surface. In a second step, the AuNPs are coated with melaniniron in a controlled way, following the electrochemical method previously described for the modification of planar $\mathrm{Au}(111)$ surfaces. ${ }^{15,16}$ Bioorganic-iron coatings have been extensively studied as efficient catalysts and electrocatalysts. In particular, iron-containing molecules, such as phthalocyanines, ${ }^{17-20}$ porphyrins, ${ }^{21-23}$ and melanins ${ }^{15,16}$ have shown promising catalytic properties for a wide range of chemical and electrochemical reactions. We show that the iron-melanin coating markedly enhances the catalytic activity of the bare AuNPs for both the hydrogen peroxide electroreduction and hydrogen evolution reaction. Our results show that electrochemistry can be used to improve nanomaterials with potential applications as efficient catalysts and electrocatalysts.

\section{Experimental}

\section{AuNP synthesis}

Thiol-capped gold nanoparticles (AuNPs) were synthesized through a modification ${ }^{24}$ of the two-phase method developed by Brust. ${ }^{11}$ After the borohydride reduction step, the nanoparticles were cleaned as follows. The organic phase was isolated, and the solvent was removed with a rotatory evaporator. The dark solid of AuNPs was suspended in absolute ethanol for $30 \mathrm{~min}$, sonicated for $1 \mathrm{~min}$ and separated from the solvent by centrifugation. The supernatant was discarded, and this washing process was repeated another three times. Further repetition of the ethanol cleaning cycle produced no change in the $\mathrm{Au}: \mathrm{S}$ ratio determined from XPS data. Finally, a small amount of acetone was added to remove the ethanol, and easily dry the AuNPs in air at room temperature.

\section{TEM characterization}

Transmission electron microscopy (TEM) characterization of thiol-capped gold nanoparticles was done using a FEI CM200 UT microscope, operating at $200 \mathrm{kV}$. Some drops of hexanedispersed nanoparticles were placed on a 400 mesh ultrathin carbon type-A copper grid (Ted-Pella) and bright-field images were taken to measure the size of the nanoparticles. The size distribution of the nanoparticles was performed using ImageJ software by measuring 200 nanoparticles without using any algorithm. ${ }^{24} \mathrm{~A}$ log-normal distribution function was fitted to the histogram obtained, according to ref. 25.

\section{Electrochemical measurements}

Electrochemical runs were made in a specially designed Teflonmade cell that leaves an exposed working electrode area of $0.28 \mathrm{~cm}^{2}$, thus eliminating the contribution of the HOPG edges in the voltammograms. $\mathrm{An} \mathrm{Ag} / \mathrm{AgCl} / \mathrm{KCl}$ saturated electrode (SSC) and a large area platinum foil were used as reference and counter electrode, respectively. ${ }^{15,16}$

\section{XPS, XANES and EXAFS characterization}

XANES measurements at the $\mathrm{Fe} \mathrm{L}_{2,3}$ edges were taken in total electron yield (TEY) mode under UHV $\left(10^{-9}\right.$ torr) at beamline
9.3.2 of Advanced Light Source at the Lawrence Berkeley National Laboratory. EXAFS measurements at the $\mathrm{Au}_{3}$ edge $(11919 \mathrm{eV})$ were performed at the XAFS-2 beam-line, LNLS (Campinas, Brazil), at room temperature in fluorescence mode using a $\mathrm{Si}(111)$ crystal monochromator. The EXAFS signals were extracted from the absorption spectra using the Athena program and analyzed using the Artemis program. ${ }^{26}$ Phase and amplitude of reference compounds used in the analysis were generated using the FEFF code. ${ }^{27}$ XPS measurements were performed with a $\mathrm{Mg} \mathrm{K} \alpha$ source (XR50, Specs $\mathrm{GmbH}$ ) and a hemispherical electron energy analyzer (PHOIBOS 100, Specs $\mathrm{GmbH}$ ). A two-point calibration of the energy scale was performed using sputtered cleaned gold (Au 4f $7 / 2$, binding energy $(\mathrm{BE})=84.00 \mathrm{eV})$ and copper $(\mathrm{Cu} 2 \mathrm{p} 3 / 2, \mathrm{BE}=932.67 \mathrm{eV})$ samples.

\section{STM and AFM imaging}

STM imaging of the nanoparticles on HOPG was made in air in the constant current mode with a STM microscope controlled by a Nanoscope IIIa unit from Veeco Instruments (Santa Barbara, CA) and by using commercial Pt-Ir tips. Typical tunneling currents, bias voltages and scan rates were $25 \mathrm{pA}, 1.5 \mathrm{~V}$, and $0.8 \mathrm{~Hz}$, respectively. The AuNPs on HOPG were also imaged by AFM operating in tapping mode using a Multimode microscope and a Nanoscope $\mathrm{V}$ control unit from Veeco Instruments at a scan rate of $1.0-1.2 \mathrm{~Hz}$. To this end, etched silicon tips (RTESP, 271-311 kHz, and 40-80 $\mathrm{N} \mathrm{m}^{-1}$, from Veeco) were used.

\section{UV/visible spectroscopy}

UV/visible spectroscopy (UV/vis) was performed with a Perkin Elmer Lambda 35 Spectrometer, equipped with a double beam. AuNPs were dispersed in hexane, which was used as reference.

\section{AuNPs immobilization, cleaning and functionalization}

The thiol-capped AuNPs were immobilized by immersing HOPG substrates in AuNP solutions in hexane, as described in ref. 14. Concentration and adsorption times were $2 \mathrm{mg} \mathrm{mL}^{-1}$ and $24 \mathrm{~h}$, respectively. For some STM and AFM imaging measurements $0.2 \mathrm{mg} \mathrm{mL}^{-1}$ solutions were used. After incubation, the AuNPs modified HOPG substrates were repeatedly rinsed with hexane and dried with nitrogen before characterization.

Bare AuNPs onto HOPG substrate were obtained by reductive electrodesorption of the thiol-capped AuNPs in $0.1 \mathrm{M} \mathrm{NaOH}$ by performing cyclic voltammetry between -0.2 and -1.7 at $0.05 \mathrm{~V} \mathrm{~s}^{-1} \cdot{ }^{14}$ Then, melanin films were electrochemically selfassembled as described in ref. 15 and 16. Briefly, the previously cleaned AuNPs supported on the HOPG surface were immersed in a synthetic eumelanin-containing aqueous $0.1 \mathrm{M} \mathrm{NaOH}$ solution $\left(0.3 \mathrm{~g} \mathrm{~L}^{-1}\right.$, melanin from Sigma, M8631). This eumelanin is formed by 5,6-dihydroxyindole (DHI) and 5,6-dihydroxyindole carboxylic acid (DHICA). The eumelanin-containing solution also has $\sim 0.3 \mathrm{ppm}$ of $\mathrm{Fe}$, as determined by atomic absorption spectroscopy. The AuNP-covered HOPG substrates were polarized at $-1.0 \mathrm{~V}$ in a conventional three-electrode electrochemical cell containing the melanin solution for $2 \mathrm{~h}$ in order to coat the nanoparticles with a melanin-iron shell. The modified 
AuNP-HOPG substrates were then removed from the cell, carefully rinsed with MilliQ water, and dried under nitrogen before characterization.

Vapor deposited Au films on glass with (111) preferred orientation (Arrandee ${ }^{\mathrm{TM}}$, AF 45 Berliner Glass KG, Germany) annealed under a hydrogen flame were used for control experiments in order to compare the behaviour of the AuNPs with that of planar Au substrates.

\section{Results and discussion}

The characterization of the thiol-capped nanoparticles is shown in Fig. 1. The UV-vis spectrum (Fig. 1a) is consistent with the presence of AuNPs with average sizes smaller than $4 \mathrm{~nm}$. In fact, the analysis of the TEM images (inset in Fig. 1b) leads to a relatively narrow particle size distribution with average size $<D>=3.0 \pm 0.9 \mathrm{~nm}$ (Fig. 1b).

XPS spectra of HOPG substrates taken after immersion in hexane solutions of thiol-capped AuNPs for $24 \mathrm{~h}$ show the typical Au 4f signal, indicating that the NPs have been efficiently adsorbed on the surface (Fig. 2a, black points). Moreover, the XPS S 2p signal with a maximum at $162 \mathrm{eV}$ (Fig. 2b, black points) reveals the presence of $\mathrm{Au}$-thiolate bonds at the NP surfaces. ${ }^{28}$ In fact, the spectrum shown in black in Fig. $2 b$ can be fitted by considering the usual three components corresponding to unbounded thiols $(163 \mathrm{eV})$, chemisorbed thiols $(162 \mathrm{eV})$ and $\mathrm{S}$ impurities $(161 \mathrm{eV}) .{ }^{28}$ However, only the contribution of chemisorbed thiols at $162 \mathrm{eV}$ was significant (the $161 \mathrm{eV}$ and $163 \mathrm{eV}$ components represented only $\sim 5 \%$ and $\sim 10 \%$, respectively).
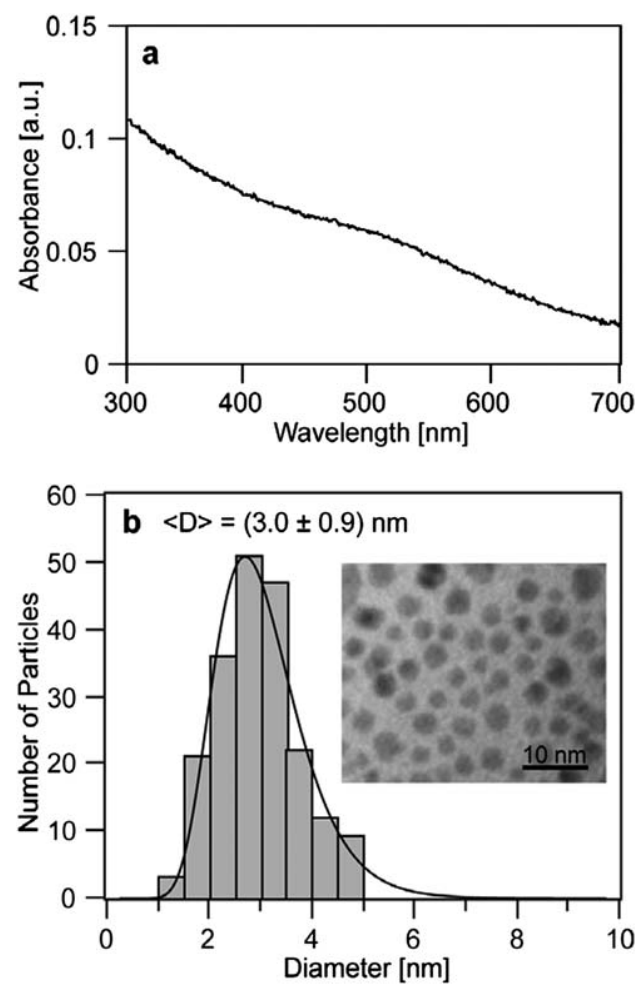

Fig. 1 (a) UV-vis spectrum of dodecanethiol-capped AuNPs in hexane. (b) Particle size distribution obtained from TEM images (inset).
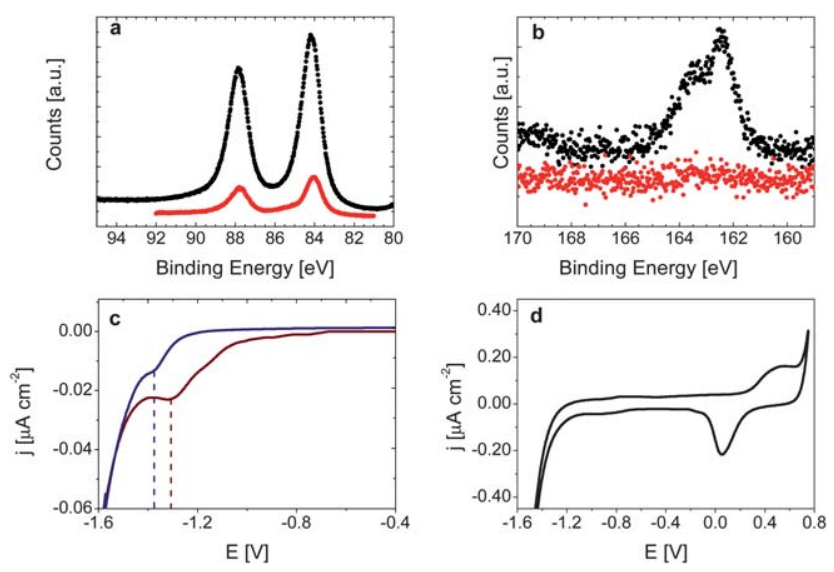

Fig. 2 (a) (Black) Au 4f XPS spectrum after immersion of HOPG in NT-capped AuNP in hexane $\left(2 \mathrm{~g} \mathrm{~L}^{-1}\right)$ for $24 \mathrm{~h}$, (red) same spectrum taken after NT reductive desorption. (b) (black) S 2p XPS spectrum for the AuNPs supported in HOPG, (red) same taken after NT reductive desorption. (c) Current density-potential profiles corresponding to reductive desorption from the NT-capped (wine line) and DT-capped (blue line) AuNPs supported on HOPG. (d) Cyclic voltammogram for the AuNPs-HOPG system after NT (or DT) reductive desorption. Scan rate $v=0.1 \mathrm{~V} \mathrm{~s}^{-1}$; electrolyte: $0.1 \mathrm{M} \mathrm{NaOH}$.

The alkanethiol capping was then removed from the nanoparticles by reductive electrodesorption by performing cyclic voltammetric scans (current density-potential profiles) between $-0.2 \mathrm{~V}$ and $-1.7 \mathrm{~V}$ at $0.05 \mathrm{~V} \mathrm{~s}^{-1}{ }^{29}$ The first scan in the negative direction shows broad cathodic humps related to the thiol reductive desorption that precedes the hydrogen evolution reaction (Fig. 2c). ${ }^{14}$ DT molecules are desorbed from the AuNPs at more negative potential values than NT ones due to the increase in the hydrocarbon chain-chain interactions. ${ }^{30}$ Moreover, the peak potentials of the desorption process are shifted in the negative direction $(\sim-0.2 \mathrm{~V})$ with respect to the corresponding thiol SAMs on $\mathrm{Au}(111)$, in agreement with previously reported data for alkanethiol desorption from AuNPs, and nanostructured (rough) Au. ${ }^{31}$ DFT calculations have shown that the binding energy of alkanethiols on Au surfaces containing defects such as steps and adatoms is higher than that found on smooth terraces, thus explaining the increase in SAM stability on the nanoparticle highly defective surfaces. ${ }^{32}$ Although several voltammetric cycles (5-6) were necessary to completely remove alkanethiol capping from the nanoparticles, data in Fig. 2c demonstrate that even alkanethiols with relatively long hydrocarbon chains can be completely removed, leading to clean AuNPs.

As already reported, ${ }^{14}$ a fraction of the AuNPs is released to the electrolyte during thiol desorption, while another fraction of the thiol-free AuNPs remains adsorbed on the HOPG surface. In fact, the XPS spectra of the clean AuNPs on the HOPG substrate (after the voltammetric runs and careful rinsing of the sample) still show the Au 4f signal (Fig. 2a, red line), while the $\mathrm{S}$ signal has disappeared (Fig. 2b, red line). This point has been verified by recording cyclic voltammograms in $0.1 \mathrm{M} \mathrm{NaOH}$ between $-1.7 \mathrm{~V}$ and $0.75 \mathrm{~V}$ for AuNP-covered HOPG samples after thiol cleaning by reductive desorption. These voltammograms clearly show the typical response of clean Au substrates (Fig. 2d), i.e. the 
formation and electroreduction of the $\mathrm{AuO}$ monolayer. This procedure allows us to estimate the surface coverage of the AuNPs on the HOPG substrate by using the charge involved in the $\mathrm{AuO}$ electroreduction. ${ }^{33}$

\section{Melanin-iron modification of clean AuNP}

The HOPG substrates modified with the thiol-free AuNPs were immersed in melanin-containing $0.1 \mathrm{M} \mathrm{NaOH}$ solutions and polarized at $-1.0 \mathrm{~V}$ for $2 \mathrm{~h}$. Afterwards, the substrates were carefully rinsed with water and inserted into the Teflon-made electrochemical cell containing $0.1 \mathrm{M} \mathrm{NaOH}$ solution. The voltammetric runs recorded between $-0.2 \mathrm{~V}$ and $-1.6 \mathrm{~V}$ at $0.1 \mathrm{~V} \mathrm{~s}^{-1}$ show two redox couples located at $-0.50 /-0.60 \mathrm{~V}(\mathrm{AI} / \mathrm{CI})$ and $-0.75 /-0.80 \mathrm{~V}$ (AII/CII) (Fig. 3a, black line). These peaks are similar to those observed for melanin-iron covered $\mathrm{Au}(111)$ surfaces and have been assigned to the quinol/quinone redox couple and $\mathrm{Fe}(\mathrm{II}) / \mathrm{Fe}$ (III) species in tetrahedral and octahedral environments, respectively ${ }^{16}$ (Fig. 3a, red line). In contrast, peaks AIII/CIII, which have been related to oxidation/reduction of co-deposited iron oxide particles species, are much smaller for the melanin-covered AuNPs than for melanin on $\mathrm{Au}(111)$.

The system has also been characterized by XPS after melanin deposition (Fig. 3b-d). First, we have verified that the Au signal arising from the AuNPs immobilized on the HOPG substrate is still present (Fig. 3b). This is not a minor point because it demonstrates that the AuNPs remain on the HOPG substrate after the long-time $(2 \mathrm{~h})$ melanin-iron deposition process. In addition to the $\mathrm{Au} 4 \mathrm{f}$ signal, the $\mathrm{N} 1 \mathrm{~s}$ and $\mathrm{Fe} 2 \mathrm{p}$ signals arising from the melanin deposit ${ }^{15,16} \mathrm{can}$ be observed (Fig. 3c and d). We have therefore compared the $\mathrm{Fe}$ and $\mathrm{N}$ signals obtained for the melanin-iron covered AuNPs with those recorded for melanin evaporated from ethanolic solutions on $\mathrm{Au}(111)$, and with those
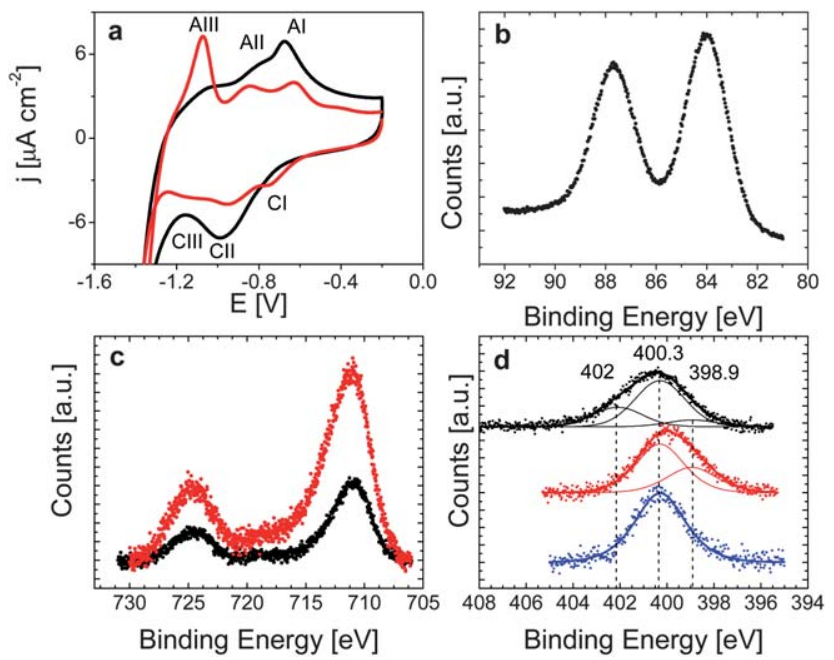

Fig. 3 (a) Voltammograms (first scan) recorded at $v=0.1 \mathrm{~V} \mathrm{~s}^{-1}$ in $0.1 \mathrm{M}$ $\mathrm{NaOH}$ for melanin-iron deposited on (black line) clean AuNPs on HOPG and (red line) Au(111). XPS spectra (b) Au 4f for melanin-iron covered AuNPs, (c) Fe 2p for:(red line) melanin-iron deposited on $\mathrm{Au}(111)$ and (black line) melanin-iron deposited on AuNPs, (d) N 1s for (black line) melanin powder evaporated on $\mathrm{Au}(111)$, (red line) melaniniron deposited on $\mathrm{Au}(111)$ and (blue line) melanin-iron deposited on clean AuNPs on HOPG. arising from melanin-iron films electrochemically deposited on preferred oriented $\mathrm{Au}(111)$ surfaces prepared following the same procedure described for AuNP modification.

While the Fe signal is not detected for evaporated melanin on $\mathrm{Au}(111)$, a fact consistent with the low iron content in the melanin powder, the Fe $2 \mathrm{p}$ signal for iron melanin electrochemically deposited both on AuNP and on preferred oriented $\mathrm{Au}(111)$ is clearly visible (Fig. 3c). This indicates that Fe-containing species are concentrated at the Au surface during the electrochemical deposition process. Moreover, XPS data indicate that $\mathrm{Fe}$ is in an oxidized state.

On the other hand, the $\mathrm{N}$ 1s peak exhibits some interesting features. In fact, the $\mathrm{N}$ 1s signal of melanin evaporated on preferred oriented $\mathrm{Au}(111)$ (Fig. 3d) can be fitted with the three components ( $399 \mathrm{eV}, 400.3 \mathrm{eV}$ and $402 \mathrm{eV}$ ) usually observed for melanins $^{34}$ (Fig. 3d, black line). While the $400.3 \mathrm{eV}$ signal has been associated to indolic $\mathrm{N}$ in DHICA monomers and dimers, ${ }^{35}$ a component at $399.4 \mathrm{eV}$ has been related to the pyrrol ring. ${ }^{36} \mathrm{In}$ the case of melanin aggregates the $400 \mathrm{eV}$ and $402 \mathrm{eV}$ components have been assigned by XAS measurements to excitations of $\pi$ and $\sigma$ orbitals, respectively. ${ }^{37}$ We note that for melanin-iron deposited on $\mathrm{Au}(111)$ only the components at $399 \mathrm{eV}$ and $400.3 \mathrm{eV}$ are significant (Fig. 3d, red line). Finally, for melanin electrochemically deposited on the AuNPs only the $\mathrm{N} 1 \mathrm{~s}$ component at $400.3 \mathrm{eV}$ is present. This means that the electrochemical method induces not only iron enrichment, but also the selective adsorption of certain melanin fractions at the surfaces; this effect being more important for the AuNPs. Therefore, they could be possibly employed for the analytical separation of oligomeric species from the complex melanin solution.

Blank experiments made using HOPG substrates free of AuNPs revealed a small amount of $\mathrm{N}$ and no evidence of $\mathrm{Fe},{ }^{38}$ i.e. no significant melanin-iron adsorption takes place on the carbon substrate during the 2 hour polarization at $-1.0 \mathrm{~V}$. Thus, the $\mathrm{N}$ and Fe signals only arise from melanin bounded to the AuNPs.

The nature of the oxidized iron present in our system was determined by XANES. Fig. 4 shows the XANES spectra at the $\mathrm{L}_{2,3}$ edges for melanin-iron AuNPs on HOPG and also for crystalline $\mathrm{Fe}_{2} \mathrm{O}_{3}$ used as reference. The $\mathrm{L}_{3}$ edge energy value of our sample is the same as that of the reference, indicating that the mean oxidation state of Fe in melanin-iron AuNPs is the same as in $\mathrm{Fe}_{2} \mathrm{O}_{3}{ }^{39}$ and in XANES data obtained for the solid melanin

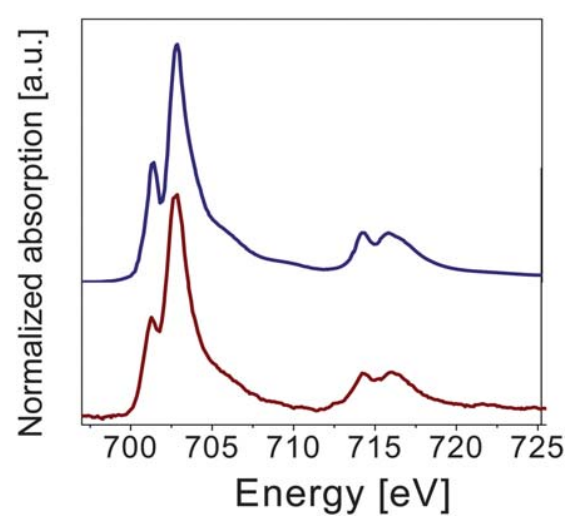

Fig. 4 XANES spectra at the $\mathrm{Fe} \mathrm{L}_{2,3}$ edges for $\mathrm{Fe}_{2} \mathrm{O}_{3}$ (blue line) and melanin-iron on AuNP (wine line). 
used as reactant in this work. ${ }^{16}$ There is also a big similarity in the general shape of both XANES spectra, showing corresponding features at both $\mathrm{L}_{2,3}$ edges.

However, previous data obtained for thick melanin-iron films on $\mathrm{Au}(111)$ indicated a more reduced state of the Fe species, close to magnetite. ${ }^{16}$ Outer $\mathrm{Fe}_{2} \mathrm{O}_{3}$-inner magnetite films are usually

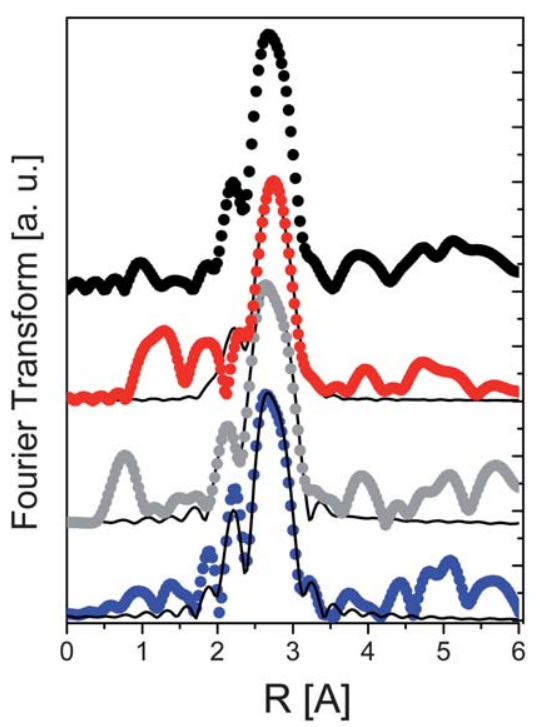

Fig. 5 Fourier Transforms of $\mathrm{Au} \mathrm{L}_{3}$ EXAFS signals corresponding to bulk Au (black circles), isolated NT-capped AuNPs in hexane (red circles), thiol-free AuNPs on HOPG (grey circles) and melanin-coveredAuNPs on HOPG (blue circles). Solid lines show the Fourier transforms of the fitted functions. observed on Fe substrates exposed to ambient conditions. ${ }^{40,41}$ Magnetite nanoparticles co-deposited with the melanin film on the $\mathrm{Au}(111)$ surface have been detected by MFM, ${ }^{16}$ and related to the voltammetric peak AIII (Fig. 3a). Both the XANES data of iron species (Fig. 4) and the absence of peak AIII in the voltammograms (Fig. 3a) indicate that the main iron component in melanin-AuNPs is Fe(III) bounded to the melanin monomers in octahedral environments. ${ }^{39}$

Fig. 5 shows the Fourier Transform of the EXAFS oscillation and the corresponding fit of the original NT-capped AuNPs in hexane, of the bare AuNPs supported on HOPG, and of the melanin-AuNP on HOPG. These results are compared to that obtained for Au films.

The fitted average coordination number $(\mathrm{ACN})$ for the $\mathrm{Au}-\mathrm{Au}$ pair for all AuNPs samples is smaller than 12, the corresponding value for the bulk Au material. The decrease in the ACN is associated with the small size of the particles. ${ }^{42}$ The ACN for the NT capped AuNP in hexane is $8.6 \pm 1$, that of the thiol-free AuNPs on HOPG is $9.5 \pm 1$ and that of the melanin-modified AuNPs on HOPG is $9.6 \pm 1$. These results indicate that, though there may be a small increase in their size after the thiol desorption, the AuNPs still retain their identity, i.e. they do not collapse by sintering and their size do not change after the melanin-iron modification.

We have also imaged the clean (thiol-free) AuNPs (Fig. 6a) and melanin-modified AuNPs on HOPG (Fig. 6b) by tapping AFM in air. In both cases the HOPG substrates are densely covered by irregular islands $50-100 \mathrm{~nm}$ in size. In the case of the clean AuNPs, most of the islands exhibit $\sim 6 \mathrm{~nm}$ in height $(h)$, although the cross-section analysis also shows islands with $h \approx 3 \mathrm{~nm}$ (Fig. 6c). These results correspond to islands formed by
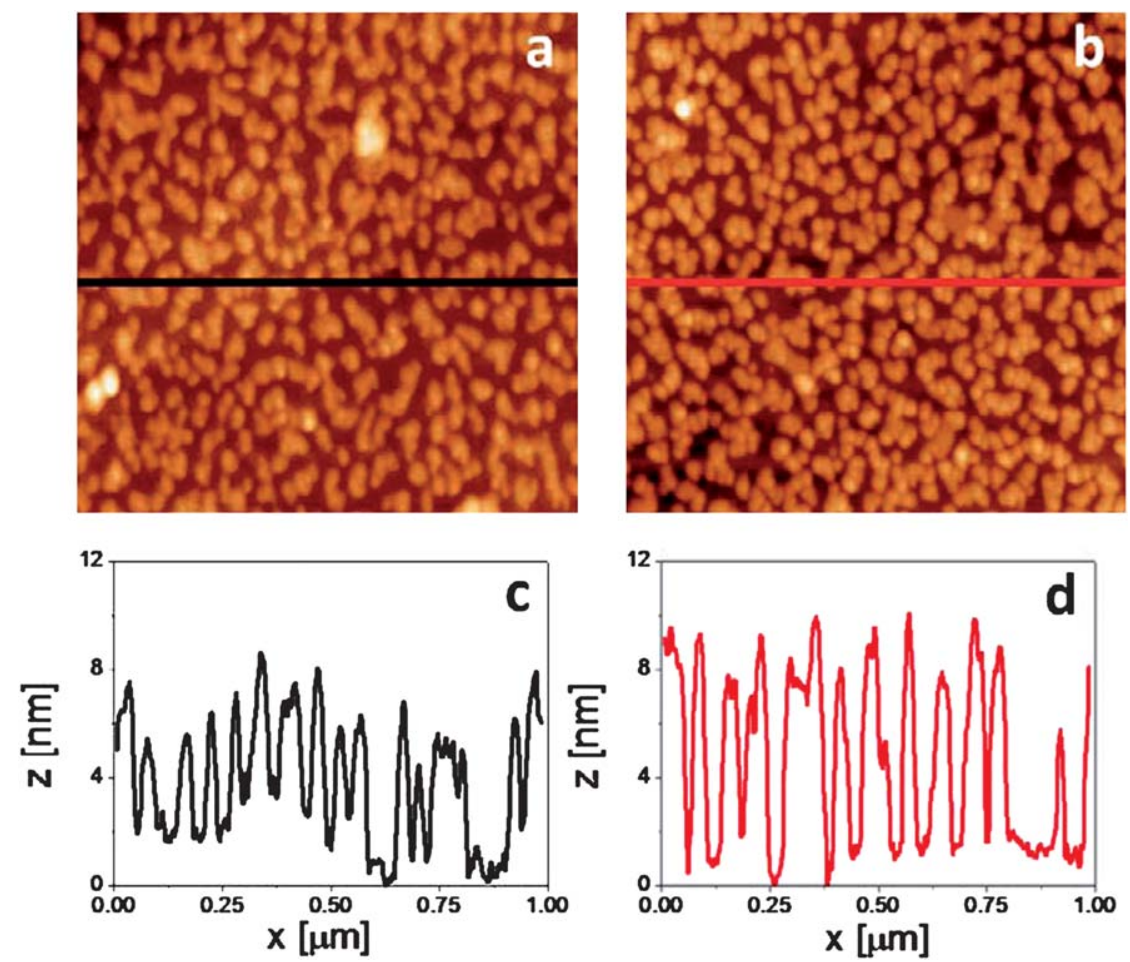

Fig. 6 In air AFM tapping images of AuNP on HOPG. $1.0 \mu \mathrm{m} \times 1.0 \mu \mathrm{m}$ top view image showing AuNP islands on HOPG (a) before and (b) after melanin-iron deposition. Cross-sections (c) before and (d) after melanin-iron deposition showing the island heights. 
Table 1 Mean height, maximum height, and root mean square roughness for islands of bare and melanin-covered AuNPs.

\begin{tabular}{lcc}
\hline & $\begin{array}{l}\text { AuNP } \\
\text { islands/nm }\end{array}$ & $\begin{array}{c}\text { Melanin-covered } \\
\text { AuNP islands/nm }\end{array}$ \\
\hline $\begin{array}{l}\text { Mean height } \\
\text { Maximum height }\end{array}$ & $5.10 \pm 0.30$ & $6.33 \pm 0.53$ \\
$\begin{array}{l}\text { Root mean square } \\
\text { roughness }\end{array}$ & $12.63 \pm 1.00$ & $16.21 \pm 2.80$ \\
& $2.37 \pm 0.23$ & $2.95 \pm 0.32$ \\
\hline
\end{tabular}

one $(h=3 \mathrm{~nm})$ and two $(h=6 \mathrm{~nm})$ layers, respectively. Fig. $6 \mathrm{~b}$ shows similar AuNP islands after melanin deposition. In this case the most frequent island height is $h \approx 8 \mathrm{~nm}$ (two AuNPs layers) although some islands formed by a single nanoparticle layer are also observed ( $h=4 \mathrm{~nm}$ ) (Fig. 6d). This means that the melaniniron coating increases the island height by $\sim 1 \mathrm{~nm}$. Table 1 shows a statistical analysis of the island heights and root mean square roughness made on several images of the substrate before and after melanin-iron deposition. All the figures indicate that melanin-iron has efficiently covered the AuNPs islands deposited on the HOPG surface. The increase in the mean height reflects the thickness of the melanin-iron coating $(\sim 1 \mathrm{~m})$, while the maximum height shows that islands composed by several AuNPs layers have also been coated by the iron-melanin film. The root mean square roughness (rms) is also interesting to confirm the increase in the AuNPs height in after melanin-iron electrodeposition.
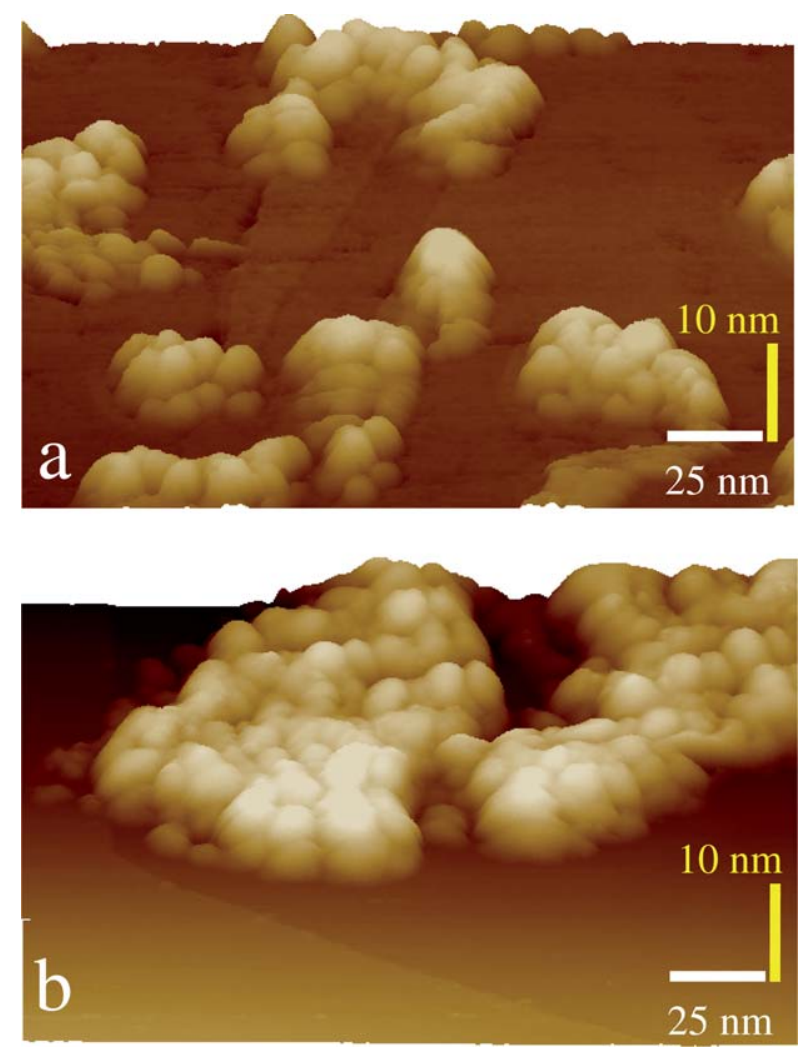

Fig. 7 STM images $(200 \mathrm{~nm} \times 165 \mathrm{~nm})$ of (a) bare AuNPs on HOPG, (b) AuNPs on HOPG after melanin-iron deposition. The white and yellow bars indicate the lateral $(x-y)$ and $z$ scales, respectively.
STM images of islands of bare (Fig. 7a) and melanin-iron covered (Fig. 7b) AuNPs are shown. The better resolution of this technique compared to AFM allows imaging the individual NPs that form the islands. The height of the first layer of AuNPs and melanin-covered AuNPs is $3-4 \mathrm{~nm}$, close to that measured by tapping AFM (Fig. 6c and d). However, here we cannot perform a statistical height analysis because it is extremely difficult to obtain noise-free STM images of the AuNP islands. In fact, although we operate our STM at very low tunneling currents, sometimes the tip "moves" the islands from one place to another. The average lateral size of the AuNPs and melanin-iron covered AuNPs (measured with the power spectral density of the software after highpass filtering, data not shown) is larger than that expected from their height: 9 and $11 \mathrm{~nm}$, respectively. We consider that this is due to tip-sample convolution that increases the real size of the nanoparticles, and also because possibly the tip cannot resolve the smallest ones, therefore showing two or three adjacent nanoparticles as just one feature.

Despite this drawback AFM, STM and EXAFS data are consistent with the presence of NPs with a melanin-iron coating $\sim 1 \mathrm{~nm}$ thick (assuming a uniform layer of melanin) on a $3 \mathrm{~nm}$ size $\mathrm{Au}$ core, thus accounting for the $1 \mathrm{~nm}$ increase in height measured by AFM (Fig. 6). Note that a $1.8 \mathrm{~nm}$ thickness has been estimated from XPS data for dopamine-melanin films on $\mathrm{Si}$ after $1 \mathrm{~h}$ immersion. ${ }^{43}$ The extremely thin melanin layer explains the presence of the Au $4 \mathrm{f}$ signal in the XPS measurements. It is interesting to note that, while metallic cations induce the formation of spherical melanin nano/micro particles, ${ }^{44}$ only extremely thin and structureless layers of melanin-iron seem to be present on the AuNPs. This is consistent with the low iron content in the coating and with the fact that synthetic eumelanin from a metal-free solution appears to be structureless.

\section{Electrocatalytic activity of AuNPs and melanin-iron covered AuNP supported on HOPG}

We have tested the catalytic ability to electroreduce hydrogen peroxide of both bare AuNPs and melanin-iron covered AuNPs supported on HOPG. The development of a biosensor for $\mathrm{H}_{2} \mathrm{O}_{2}$ involves important areas of application, like clinical, food and pharmaceutical industries, and also environmental analyses. ${ }^{45}$ Moreover, hydrogen peroxide is also used as an antibacterial agent in the food and beverage industry. These applications have raised extensive demands for establishing protocols for $\mathrm{H}_{2} \mathrm{O}_{2}$ detection. Additionally, $\mathrm{H}_{2} \mathrm{O}_{2}$ is an important analyte because it is one of the most important products/substrates of enzyme catalyzed oxidation reactions. ${ }^{45}$ The $\mathrm{H}_{2} \mathrm{O}_{2}$ electroreduction reaction yields $\mathrm{OH}^{\cdot}$ and $\mathrm{HO}^{-}$species. ${ }^{16}$

In Fig. 8 the cathodic polarization curves recorded for $\mathrm{HOPG}$, $\mathrm{Au}(111)$, bare AuNPs on HOPG, and melanin-iron covered AuNPs on HOPG in $4 \mathrm{mM} \mathrm{H}_{2} \mathrm{O}_{2}+0.1 \mathrm{M} \mathrm{NaOH}$ are shown. The total surface coverage of AuNPs in these experiments was less than 0.1 (determined from gold oxide electroreduction experiments). While no significant electroreduction of hydrogen peroxide takes place on the plain HOPG substrate (Fig. 8, black line), a cathodic current peak at $-1.15 \mathrm{~V}$, related to hydrogen peroxide electroreduction, preceding the hydrogen evolution reaction (HER) is seen for $\mathrm{Au}(111)$ (Fig. 8, red line). However, the catalytic activity for the hydrogen peroxide electroreduction 


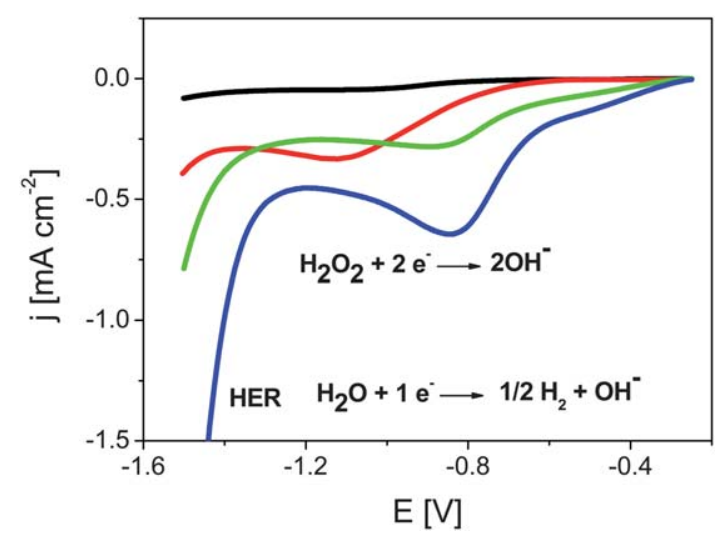

Fig. 8 Cathodic polarization curves recorded at $0.025 \mathrm{~V} \mathrm{~s}^{-1}$ in $4 \mathrm{mM}$ $\mathrm{H}_{2} \mathrm{O}_{2}+0.1 \mathrm{M} \mathrm{NaOH}$. (black) HOPG, (red) $\mathrm{Au}(111)$, (green) clean AuNPs on HOPG, (blue) melanin-iron covered AuNP (deposition time $=120 \mathrm{~min}$ ). The current density $(j)$ is referred to the geometrical area of the HOPG substrate.

is rather poor, involving large overpotentials. In fact, it is wellknown that gold is not a suitable catalyst for this reaction, in particular the $\mathrm{Au}(111)$ face. ${ }^{46}$ On the other hand, the presence of AuNPs has a considerable effect, turning the inert HOPG into an electrocatalytically active substrate with a significant hydrogen peroxide electroreduction current peak at $-0.9 \mathrm{~V}$ (Fig. 8, green line). Interestingly, the AuNP-modified HOPG substrates are much more active than clean $\mathrm{Au}(111)$ surfaces (Fig. 8, red line), reducing the overpotential in $0.25 \mathrm{~V}$. It has been shown that hydrogen peroxide electroreduction takes place on $\mathrm{Pt}$ by $\mathrm{HO}-\mathrm{OH}$ adsorption with the oxygen atom pointed out from the metal surface. Adsorption is followed by elongation and $\mathrm{O}-\mathrm{O}$ bond scission, resulting in $\mathrm{Pt}-\mathrm{OH}$ formation. ${ }^{46}$ In contrast, a negligible elongation takes place on $\mathrm{Au}(111)$ or $\mathrm{Au}(110)$ faces, explaining their poor catalytic behavior.

On the other hand, it has been reported that $\mathrm{O}-\mathrm{O}$ interactions on $2 \mathrm{~nm}$ AuNPs are quite different from those corresponding to flat $\mathrm{Au}(111)$ surfaces because low-coordinated $\mathrm{Au}$ atoms enable strong $\mathrm{O}-\mathrm{O}$ adsorption, although this is not enough to explain $\mathrm{O}-\mathrm{O}$ dissociation. ${ }^{47}$ The minimum energy pathway occurs on (100) facets with $\mathrm{O}-\mathrm{O}$ at bridge sites. In this case elongation of the $\mathrm{O}-\mathrm{O}$ bond leading to dissociation is predicted, but this effect is restricted to AuNPs smaller than 55 atoms. ${ }^{47,48}$

Our results demonstrate that $3 \mathrm{~nm}$ AuNPs are very active for hydrogen peroxide electroreduction, although we cannot exclude that the catalytic activity arises from the smallest particles $(<2 \mathrm{~nm})$ observed in the size distribution function (Fig. 1b). ${ }^{49}$

On the other hand, the iron-melanin covered AuNPs (Fig. 8, blue line) exhibit the highest catalytic activity, with a peak potential of $-0.82 \mathrm{~V}$ and the largest current density in the overall potential range. Note that the electrocatalytic effect is observed in the potential range where the melanin-iron films are electrochemically active (peaks CI and CII in Fig. 3a). Therefore, a small amount of melanin-iron on AuNPs reduces the overpotential needed for $\mathrm{H}_{2} \mathrm{O}_{2}$ reduction with respect to the AuNPs and has been assigned to an enhanced electron transfer from the $\mathrm{H}_{2} \mathrm{O}_{2}$ to the $\mathrm{Au}(111)$ surface mediated by the quinol/quinone groups and the $\mathrm{Fe}(\mathrm{II}) / \mathrm{Fe}(\mathrm{III})$ system as discussed in ref. 16.

Finally, we also note that the HER reaction is enhanced on the AuNP-modified HOPG, in particular in the case of melanin-iron

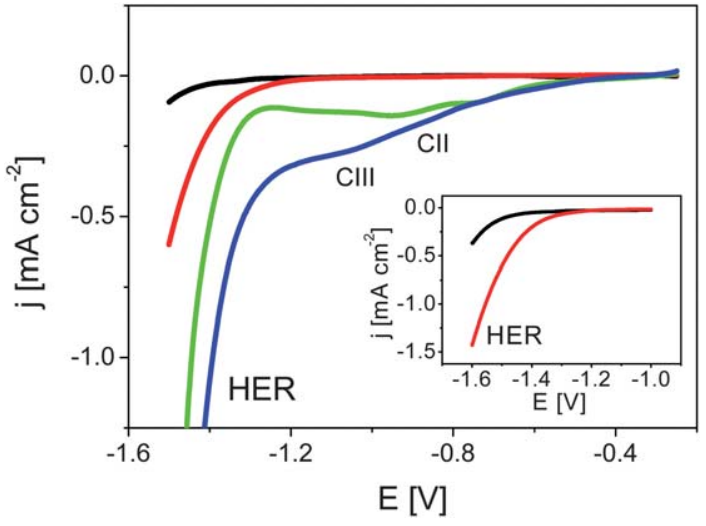

Fig. 9 Cathodic polarization curves recorded at $0.025 \mathrm{~V} \mathrm{~s}^{-1}$ in $0.1 \mathrm{M}$ $\mathrm{NaOH}$. (black) Au(111), (red) clean AuNPs on HOPG, (green) melaniniron covered $\mathrm{Au}(111)$ (deposition time $120 \mathrm{~min}$ ), (blue) melanin-iron covered AuNPs on HOPG (deposition time $120 \mathrm{~min}$ ). The current density $(j)$ is referred to the geometrical area of the HOPG substrate. The inset shows in more detail the HER reaction on (black) $\mathrm{Au}(111)$ and (red) clean AuNPs.

covered AuNPs. The HER on different Au substrates has been explored in more detail in oxygen-free $0.1 \mathrm{M} \mathrm{NaOH}$, as shown in Fig. 9. In effect, we observe that the bare AuNPs exhibit an enhancement of the HER in this electrolyte with respect to the $\mathrm{Au}(111)$ surface (Fig. 9 and inset, red $v s$. black lines). The surface of the AuNPs could provide a larger number of free electroactive sites for the adsorption of water molecules present in the alkaline medium that are catalytically reduced to hydrogen by applying the adequate potential, with a resulting increment in the rate of the hydrogen evolution reaction. ${ }^{50}$ Water dissociation on Au and $\mathrm{Cu}$ nanoparticles is associated with the low-coordinated corners and the edge sites, as well as with the fluxionality of the particles, which makes the nanoparticles more active than flat surfaces for breaking the $\mathrm{O}-\mathrm{H}$ bond. ${ }^{51}$

More interestingly, the melanin-iron coatings further improves HER for both melanin-iron covered $\mathrm{Au}(111)$ and melanin-iron covered AuNPs (Fig. 9, green and blue lines). In particular, the melanin-iron covered AuNPs exhibit the largest current densities for all the potential range. Note that the enhanced HER in Fig. 9 is preceded by the two cathodic waves at $\sim-1.0 \mathrm{~V}$ corresponding to the CII-CIII peaks, related to the $\mathrm{Fe}(\mathrm{II})$ to $\mathrm{Fe}(0)$ redox couples (see also Fig. 3a). It is well known that hydrogenase and its analogues include both a protonacceptor (a negatively charged non-metal site) to strongly trap the protons, and a hydride-acceptor (usually the highly coordinated and isolated metal site) to provide moderate bonding to the hydrogen.

Surfaces with the cooperative function of these two kinds of sites display a high catalytic activity in the HER region. Therefore, both negatively and positively charged sites are needed in the surface for catalysis (ensemble effect). ${ }^{52}$ The Fe environment in melanins could fulfill this requirement because it consists of six oxygens in a structurally distorted octahedral arrangement with a Fe-O bond length of $0.199 \mathrm{~nm} .{ }^{39}$ For instance, Fe porphyrins, with similar geometry and a Fe-N bond length of $0.2 \mathrm{~nm},{ }^{53}$ exhibit catalytic activity for the $\mathrm{HER}^{22}$ which has been related to the porphyrin- $\mathrm{Fe}(0)$ formed at negative potential values by 
reduction of the porphyrin-Fe(II) species. We propose that the catalytic activity observed in the melanin-iron system involves water reduction to $\mathrm{H}_{2}$ and $\mathrm{OH}^{-}$and the oxidation of melanin$\mathrm{Fe}(0)$ to melanin-Fe(II). The melanin-Fe(II) is then reduced to melanin-Fe(0) at these high negative potential values, thus allowing a new reaction cycle.

\section{Conclusions and outlooks}

Reductive electrodesorption has been used to produce "naked" gold nanoparticles $3 \mathrm{~nm}$ in size (AuNPs) on HOPG from thiolcapped AuNPs. The clean AuNPs transform the electrocatalytic inert HOPG surface into an active surface for the hydrogen peroxide electroreduction, lowering the overpotential $\sim 0.2 \mathrm{~V}$ with respect to the $\mathrm{Au}(111)$ surface. The nanoparticles exhibit also an increase in the hydrogen evolution reaction with respect to the same substrate. In a second step a melanin-iron shell has been electrochemically formed around the AuNPs. This procedure results in melanin-iron covered AuNPs supported on the carbon surface. The melanin-iron ultrathin coating largely improves the catalytic activity of the bare AuNPs for both hydrogen peroxide electroreduction and hydrogen evolution reaction. This strategy, which integrates electrochemistry to nanomaterials, can be applied to the preparation of efficient "naked" AuNP and Fe-organic capped AuNP catalysts.

\section{Acknowledgements}

We acknowledge financial support from ANPCyT (PICT06-621, PICT05-632439, PICT-2008-00038), CONICET (CONICET (PIP 11220090100139 and PIP 11220080103079) and UNLP, Argentina, CTQ2008-06017/BQU, Spain and ID20100152, ACIISI, Gobierno de Canarias and LNLS, Brazil under project D04B-XAFS1-7153. AGO acknowledges MICINN for a FPU fellowship, Spain. This paper was made in the frame of the Interfacial, Supramolecular and Molecular Nanoscience and Nanotechnology Net and Nanoscience (PAE, 22771, ANPCyT). The authors thankfully acknowledge Eugenia Zelaya for assistance in TEM analysis.

\section{References}

1 U. Drechsler, B. Erdogan and V. M. Rotello, Chem.-Eur. J., 2004, 10, 5570-5579.

2 G. Merga, N. Saucedo, L. C. Cass, J. Puthussery and D. Meisel, J. Phys. Chem. C, 2010, 114, 14811-14818.

3 M. Haruta, Catal. Today, 1997, 36, 153-166.

4 M. Haruta, Gold Bull., 2004, 37, 27-36.

5 M. Haruta, S. Tsubota, T. Kobayashi, H. Kageyama, M. J. Genet and B. Delmon, J. Catal., 1993, 144, 175-192.

6 M. Haruta, N. Yamada, T. Kobayashi and S. Iijima, J. Catal., 1989, 115, 301-309.

7 S. Peng, Y. M. Lee, C. Wang, H. F. Yin, S. Dai and S. H. Sun, Nano Res., 2008, 1, 229-234.

8 H. G. Zhu, C. D. Liang, W. F. Yan, S. H. Overbury and S. Dai, J. Phys. Chem. B, 2006, 110, 10842-10848.

9 G. R. Bamwenda, S. Tsubota, T. Nakamura and M. Haruta, Catal. Lett., 1997, 44, 83-87.

10 J. Chou, N. R. Franklin, S. H. Baeck, T. F. Jaramillo and E. W. McFarland, Catal. Lett., 2004, 95, 107-111.

11 M. Brust, M. Walker, D. Bethell, D. J. Schiffrin and R. Whyman, J. Chem. Soc., Chem. Commun., 1994, 801-802.

12 P. D. Jadzinsky, G. Calero, C. J. Ackerson, D. A. Bushnell and R. D. Kornberg, Science, 2007, 318, 430-433.
13 C. Vericat, M. E. Vela, G. Benitez, P. Carro and R. C. Salvarezza, Chem. Soc. Rev., 2010, 39, 1805-1834.

14 D. Grumelli, C. Vericat, G. Benitez, M. E. Vela, R. C. Salvarezza, L. J. Giovanetti, J. M. Ramallo-Lopez, F. G. Requejo, A. F. Craievich and Y. S. Shon, J. Phys. Chem. C, 2007, 111, 71797184.

15 P. Diaz, Y. Gimeno, P. Carro, S. Gonzalez, P. L. Schilardi, G. Benitez, R. C. Salvarezza and A. H. Creus, Langmuir, 2005, 21, 5924-5930.

16 A. G. Orive, P. Dip, Y. Gimeno, P. Díaz, P. Carro, A. H. Creus, G. Benítez, P. L. Schilardi, L. Andrini, F. Requejo and R. C. Salvarezza, Chem.-Eur. J., 2007, 13, 473-482.

17 F. Vandenbrink, W. Visscher and E. Barendrecht, J. Electroanal. Chem., 1984, 175, 279-289.

18 F. Vandenbrink, W. Visscher and E. Barendrecht, J. Electroanal. Chem., 1984, 172, 301-325.

19 K. I. Ozoemena and T. Nyokong, Talanta, 2005, 67, 162-168.

20 O. L. Kaliya, E. A. Lukyanets and G. N. Vorozhtsov, J. Porphyrins Phthalocyanines, 1999, 3, 592-610.

21 Y. O. Su, T. Kuwana and S. M. Chen, J. Electroanal. Chem., 1990, 288, 177-195.

22 I. Bhugun, D. Lexa and J. M. Saveant, J. Am. Chem. Soc., 1996, 118, 3982-3983.

23 J. P. Collman, M. Kaplun, C. J. Sunderland and R. Boulatov, J. Am. Chem. Soc., 2004, 126, 11166-11167.

24 M. D. Abramoff, P. J. Magelhaes and S. J. Ram, Biophotonics Int., 2004, 11, 36-42.

25 C. G. Granqvist and R. A. Buhrman, J. Appl. Phys., 1976, 47, 22002219.

26 B. Ravel and M. Newville, J. Synchrotron Radiat., 2005, 12, 537541.

27 S. I. Zabinsky, J. J. Rehr, A. Ankudinov, R. C. Albers and M. J. Eller, Phys. Rev. B: Condens. Matter Mater. Phys., 1995, 52, 2995-3009.

28 C. J. Zhong, R. C. Brush, J. Anderegg and M. D. Porter, Langmuir, $1999,15,518-525$.

29 C. Vericat, G. Andreasen, M. E. Vela and R. C. Salvarezza, J. Phys. Chem. B, 2000, 104, 302-307.

30 M. E. Vela, H. Martin, C. Vericat, G. Andreasen, A. H. Creus and R. C. Salvarezza, J. Phys. Chem. B, 2000, 104, 1187811882.

31 E. Cortes, A. A. Rubert, G. Benitez, P. Carro, M. E. Vela and R. C. Salvarezza, Langmuir, 2009, 25, 5661-5666.

32 J. C. Love, L. A. Estroff, J. K. Kriebel, R. G. Nuzzo and G. M. Whitesides, Chem. Rev., 2005, 105, 1103-1169.

33 R. C. Salvarezza and A. J. C. Arvia, in Modern Aspects of Electrochemistry, ed. B. E. Conway, J. O. M. Bockris and R. E. White, Plenum Press, New York, 1996, vol. 28, ch. 5, pp. 289-373.

34 M. B. Clark, J. A. Gardella, T. M. Schultz, D. G. Patil and L. Salvati, Anal. Chem., 1990, 62, 949-956.

35 M. L. Tran, B. J. Powell and P. Meredith, Biophys. J., 2006, 90, $743-$ 752.

36 C. Malitesta, I. Losito, L. Sabbatini and P. G. Zambonin, J. Electron Spectrosc. Relat. Phenom., 1995, 76, 629-634.

37 L. Sangaletti, S. Pagliara, P. Vilmercati, C. Castellarin-Cudia, P. Borghetti, P. Galinetto, R. Gebauer and A. Goldoni, J. Phys. Chem. B, 2007, 111, 5372-5376.

38 G. A. Orive, Y. Gimeno, A. Hernández Creus, D. Grumelli, C. Vericat, G. Benitez and R. C. Salvarezza, Electrochim. Acta, 2007, 54, 1589-1596.

39 A. J. Kropf, B. A. Bunker, M. Eisner, S. C. Moss, L. Zecca, A. Stroppolo and P. R. Crippa, Biophys. J., 1998, 75, 31353142 .

40 V. Goossens, J. Wielant, S. Van Gils, R. Finsy and H. Terryn, Surf. Interface Anal., 2006, 38, 489-493.

41 U. Schwertmann and R. M. Cornell, Iron Oxides in the Laboratory, Wiley VCH, Weinheim, 2000.

42 M. A. Ramallo-Lopez, F. G. Requejo, A. F. Craievich, J. Wei, M. Avalos-Borja and E. Iglesia, J. Mol. Catal. A: Chem., 2005, 228, 299-307.

43 F. Bernsmann, A. Ponche, C. Ringwald, J. Hemmerle, J. Raya, B. Bechinger, J. C. Voegel, P. Schaaf and V. Ball, J. Phys. Chem. C, 2009, 113, 8234-8242.

44 P. Borghetti, A. Goldoni, C. Castellarin-Cudia, F. H. Casalis, L. Floreano, A. Cossaro, A. Verdini, R. Gebauer, P. t. Ghosh and L. Sangaletti, Langmuir, 2010, 26, 19007-19013. 
45 L. Giannoudi, E. V. Piletska and S. A. Piletsky, in Biotechnological Applications of Photosynthetic Proteins: Biochips, Biosensors and Biodevices, ed. M. T. Giardi and E. V. Piletska, Springer, New York, 2006, ch. 4

46 X. Li, D. Heryadi and A. A. Gewirth, Langmuir, 2005, 21, 92519259.

47 A. Roldan, S. Gonzalez, J. M. Ricart and F. Illas, ChemPhysChem, 2009, 10, 348-351.

48 L. Barrio, P. Liu, J. A. Rodriguez, J. M. Campos-Martin and J. L. G. Fierro, J. Phys. Chem. C, 2007, 111, 1900119008.
49 M. Turner, V. B. Golovko, O. P. H. Vaughan, P. Abdulkin, A. Berenguer-Murcia, M. S. Tikhov, B. F. G. Johnson and R. M. Lambert, Nature, 2008, 454, 981-U931.

50 M. Maltez-da Costa, A. de la Escosura-Muñiz and A. Merkoçia, Electrochem. Commun., 2010, 12, 1501-1504.

51 P. Liu and J. A. Rodriguez, J. Chem. Phys., 2007, 126, 164705164708 .

52 J. A. Rodriguez, P. Liu, in New Developments in Quantum Chemistry, ed. J. L. Paz and A. J. Hernández, 2008, p. 135.

53 J. P. Collman, R. R. Gagne, C. A. Reed, W. T. Robinson and G. A. Rodley, Proc. Nat. Acad. Sci. U. S. A., 1974, 71, 1326-1329. 